\title{
Erosive-thermal transition in high-flux focused ion beam nanomachining of surfaces
}

\author{
K. Das, ${ }^{a}$ J. B. Freund, ${ }^{\mathrm{a}, \mathrm{b}}$ and H. T. Johnson ${ }^{\mathrm{a}}$ \\ ${ }^{a}$ Department of Mechanical Science and Engineering \\ University of Illinois at Urbana-Champaign \\ 1206 W. Green St. \\ Urbana, IL 61801 \\ ${ }^{\mathrm{b}}$ Department of Aerospace Engineering \\ University of Illinois at Urbana-Champaign \\ 104 S. Wright St. \\ Urbana, IL 61801
}

\begin{abstract}
Focused ion beams (FIB) are increasingly used for surface modification and fabrication with nanometer scale precision. In FIB, an energetic beam of ions strikes a surface and removes material, a process that is understood to depend upon the properties of the beam (e.g. beam flux, ion energy) and is thought to be due to ion induced sputter erosion. We show that the material removal rate is also strongly affected by the thermal properties of the material, sample temperature, and geometry. Furthermore, we deduce a dimensionless parameter, a ratio of incident power to thermally dissipated power $\left(Q_{F I B}\right)$, which parameterizes a switch of the underlying mechanism of material removal. It predicts with remarkable accuracy a previously overlooked transition from slow erosive material removal to significantly accelerated thermal vaporization material removal. Its critical value explains an observed transition in data covering a range of beam fluxes, ion energies, spot sizes, film thicknesses, materials, ion species, and temperatures. Large-scale parallel molecular dynamics simulations support this transition.
\end{abstract}


Ion beam flux, defined as the number of ions incident on a surface per unit area per unit time, is a fundamental parameter in FIB material processing. A corresponding beam current is easily computed from the flux, since each incident ion typically brings one charge to the surface, though electrostatic or charge neutralization effects might alter any direct correspondence. Thus, with the beam flux (or the current) and the nominal sputter yield (the number of sputtered atoms per incident ion, typically near 1) it is simple to estimate a material removal rate during FIB processing. For example, for a widely studied material like silicon, with typical ion energies $(30 \mathrm{keV}-50 \mathrm{keV})$ and currents estimated from reported beam spot size (typically $<1 \mathrm{pA})$, the expected sputter yield $(\mathrm{SY} \approx 2.0)^{1}$ yields the independently measured erosion rates in table $\mathrm{I}^{2-5}$

However, several recent studies report material removal rates that are orders of magnitude higher than such estimates. Table I presents results for a variety of conditions: energies from 15 to 200 $\mathrm{keV}$; beam currents ranging over three orders of magnitude; target materials including metals, semiconductors, and polymers; various dwell times, pixel sizes, and ion beam species. In many cases, the corresponding estimate of number of incident ions required to remove the observed volume of atoms is a factor of $\sim 10^{3}$ larger than the actual number of ions delivered. We describe this phenomenon with a parameter called normalized material removal rate $\left(R_{M R}\right)$ representing the ratio of the observed volume of material removal to the expected volume of material removal based on a sputtering mechanism.

Various explanations have been proposed for these anomalous results. Matovic et al. ${ }^{6}$ suggest that the high sputter yield they deduce is due to the small thickness of the film, which renders the system inefficient in dissipating heat from the impact zone. As a result, the temperature within the zone is expected to significantly exceed the melting point. For sufficiently high flux, they claim that a hole is created via evaporation.

Liu et al. ${ }^{9}$ report FIB material removal that is more than $10^{3}$ faster than that predicted by sputter yield for PMMA membranes. They speculate that the anomalous sputter yield, of $10^{4}$ atoms/ion by their own estimate, is due to an ion-enhanced degradation mechanism, an unzipping reaction which, without ion beam irradiation, would only be observed at high temperatures. 
Kolibal et al., ${ }^{2}$ Hopman et al., ${ }^{3}$ and Li et al. ${ }^{4}$ study the dependence of enhanced material removal rate of $\mathrm{Si}$ on dwell time ( $1 \mu \mathrm{s}$ to $5 \mathrm{~ms})$ and beam energy ( $5 \mathrm{keV}$ to $30 \mathrm{keV})$. They observe increased material removal rate and report that the discrepancy depends on the beam parameters, namely increased dwell time and ion beam energy.

Particularly relevant to the present results, $\mathrm{Chu}$ et $a l^{7,11}$ measure the temperature rise in a $300 \mathrm{~mm}$ thick, $40 \mu \mathrm{m}$ wide PMMA substrate, due to two experiments with a $15 \mathrm{kV}$ electron beam: one with FWHM $1.7 \mu \mathrm{m}$ and $150 \mathrm{nA}$ and the other with $2.0 \mu \mathrm{m}$ and $600 \mathrm{nA}$. They report an $18 \mathrm{~K}$ temperature increase of the substrate after $100 \mu \mathrm{s}$. If we consider the electron beam or ion beam as a localized thermal source, and for simplicity assume constant bulk thermal properties of the material, our continuum finite element simulation $^{12}$ suggests that such a temperature increase corresponds to a local target temperature of nearly $9000 \mathrm{~K}$ at the center of the beam over a $400 \mathrm{~nm}$ spot. While obviously unphysical, this does suggest that an extreme thermal effect may be responsible for the anomalous material removal rate.

Similar temperature distributions are calculated by Schmied et al., ${ }^{13}$ who model spatial temperature evolution in HDPE (high density polyethylene), PMMA, and silicon due to FIB processing. They use SRIM to estimate ion energy deposition and energy dissipation to phonons, along with calculations based on a thermal spike model. ${ }^{14}$ Using this model, they simulate a $30 \mathrm{keV}$ Ga ion beam with 40nm FWHM and 500pA beam current, parameters readily achieved using commercial FIB instruments. In this case they find a peak temperature of $6300 \mathrm{~K}$ for PMMA, 2400K for HDPE, and 400K for Si. They also perform FIB experiments with a $30 \mathrm{keV}$

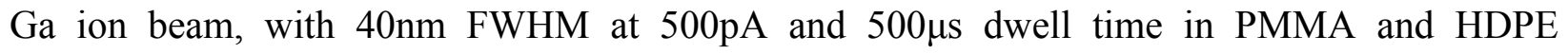
materials. The material volume removed by the FIB process in the milled structures matches well with their thermal system predictions.

Orthacker et al. report similar FIB experiments in a range of soft materials. ${ }^{15}$ They observe an enhanced material removal rate (factor of $\sim 10^{3}$ ) when the dwell time is increased or when the distance between two neighboring target pixels is decreased. Although observed material removal rates in silicon tend to be much lower than in soft materials, increased dwell time also enhances the rates in silicon. ${ }^{2,3}$ Indeed, increasing dwell time or decreasing point pitch increases the temperature near the ion beam impact zone significantly, leading to volatizing effects as 
reported by Orthacker et al. ${ }^{15}$ This evidence points to the possibility that the accelerated material removal may be due to a thermal loading condition.

Molecular dynamics simulations of FIB bombardment of silicon provide a microscopic model of FIB mechanisms. Our results, summarized below, show in detail that for increasing beam flux, material removal suddenly changes from a sputter erosion driven process at lower fluxes to a thermally driven process at higher fluxes. ${ }^{16}$

Together, these studies suggest an orders-of-magnitude variability in material removal rates depending on the beam current, beam energy, material properties, and other parameters. Low thermal conductivity materials are more susceptible to large temperature increases. Similarly, enhanced material removal is often observed in thin membranes, and for longer beam dwell times and denser target pixel distributions.

Target properties and the specifics of the FIB instrument, shown schematically in Figure 1, both affect material removal. Parameters include ion beam energy ( $E$, typically measured in $\mathrm{keV}$ ), time between impacts ( $\delta t$, in picoseconds), ion beam current ( $I$, in Amperes), accelerating potential $\left(V\right.$, in Volts), ion beam flux $\left(\varphi\right.$, ions $\left./ \mathrm{m}^{2} / \mathrm{s}\right)$, target material thermal conductivity $\left(k, \mathrm{Wm}^{-}\right.$ ${ }^{1} \mathrm{~K}^{-1}$ ), target layer thickness ( $h$, in nanometers), penetration depth ( $p$, in nanometers), FWHM diameter of the beam ( $d$, in nanometers), and a characteristic temperature difference $(\Delta T$, in Kelvins). Here we define $\Delta T=T_{b}-T_{s}$, where $T_{\mathrm{b}}$ is the boiling temperature of the material, and $T_{\mathrm{s}}$ is the target temperature. Using these we construct a dimensionless number to anticipate a thermal threshold behavior in the material removal rate.

The amount of energy deposited in the target depends on the ion penetration depth, $p$, and the target thickness, $h$. One can define an energy deposition function $g(h, p)$, or the fraction of ion energy deposited in the target, to account for this effect. For example, SRIM estimates of ion beam energy deposition with respect to depth into the target suggest that ion beams deposit approximately $1 \mathrm{keV}$ energy for every $1 \mathrm{~nm}$ target thickness. Thus we may take

$$
\begin{aligned}
& g(h, p)=1, \text { if } h>p \\
& g(h, p)=\frac{h}{p}, \text { if } h<p
\end{aligned}
$$


That is, a $30 \mathrm{keV}$ ion beam will deposit all of its energy if the target thickness is $30 \mathrm{~nm}$ or higher, and deposit a fraction of it if the target thickness is smaller than $30 \mathrm{~nm}$. One can obviously construct a more detailed relation, based for example on specific predicted behaviors for specific materials, but we shall see that this linear relation is sufficient for present purposes. Regardless of the form of $g(h, p)$, the power input can be defined as

$$
P_{\text {in }}=g(h, p) E \phi \frac{\pi d^{2}}{4}
$$

We define the lateral cylindrical surface area $A=\pi h d$ as characteristic for thermal conduction out of the target region, with a characteristic length $l_{o}=d$. The power conduction away from the impact zone is thus approximately

$$
P_{\text {out }} \approx \frac{k A \Delta T}{l_{o}}=\frac{k \pi h d \Delta T}{d}=k \pi h \Delta T
$$

Our interest is whether or not $P_{\text {out }}$ can compensate for $P_{\text {in }}$ due to the beam. That is, if the target zone, at $T_{\mathrm{b}}$, can dissipate energy by thermal conduction rapidly enough, then rapid vaporization and material removal is not be expected, and slow sputter erosion is the only mass removal mechanism. Thus we define the relevant dimensionless parameter $Q_{F I B}$ as the ratio of the incident power to the outgoing power, or

$$
Q_{F I B}=\frac{P_{\text {in }}}{P_{\text {out }}}=\frac{g(h, p) E \phi \frac{\pi d^{2}}{4}}{k \pi h \Delta T}=\frac{g(h, p) E \phi d^{2}}{4 k h \Delta T}
$$

Table II shows $Q_{F I B}$ for multiple experimental studies, including the studies represented in Table I for which the necessary information is available to evaluate this ratio.

Despite the variety of parameters involved, $Q_{F I B} \approx 1$ demarcates a clear threshold between slow and rapid material removal, as shown in Figure 2. The data divide very obviously into two regimes. None of the individual parameters, including beam current for example, which is shown in color, indicate such a threshold. There are multiple materials on either side of the apparent threshold, but no single material appears on both sides of the threshold, at least among the experimental data that are available. There are several possible explanations for this, 
including the fact that the thermal properties of different materials can be different by orders of magnitude, so that the QFIB parameter can be similarly disparate. It is also possible that in processing different materials, one is interested in achieving different effects (e.g. surface smoothing, deep etching, etc.) so that certain materials tend to be processed in different ways than other materials. Nevertheless, the presence of the apparent threshold as seen in the experimental data suggests that it may be possible to tune experimental conditions in such a way that a single material could fall either above or below the threshold.

In view of this analysis, one can predict that if other parameters are fixed, increasing the beam current (proportional to ion flux) should have a similar effect as reducing the thermal conductivity or reducing the film thickness. This prediction is supported by our computational results for silicon, which is easily modeled via molecular dynamics using accurate, wellestablished potentials. In such a calculation, we vary the ion beam current from $27 \mathrm{nA}$ to $320 \mathrm{nA}$ on two targets with $11 \mathrm{~nm}$ and $55 \mathrm{~nm}$ thickness. While some variation in beam shape is possible, we assume here for simplicity that the beam profile is Gaussian. A $3.5 \mathrm{~nm}$ FWHM, 50keV ion beam is used for the $11 \mathrm{~nm}$ thick target, and a $30 \mathrm{keV}$ ion beam is used on the $55 \mathrm{~nm}$ thick target, yielding values of the dimensionless number ranging from approximately 0.1 to 5 . Complete details of these calculations are reported elsewhere. ${ }^{16,18}$ The results of these calculations, shown in Figure 3 along with the experimental data, not only fit the clear trend and sharp threshold seen experimentally, but they make it possible to characterize the nature of the thermal effect when the incident power overwhelms the system capacity for thermal dissipation. Indeed, we see evidence of explosive boiling, including bubble formation, coalescence, and rupture, as part of the rapid fluctuations in thermodynamic state of the material near the incident ion beam. ${ }^{18}$ The computational results span a relatively small range in QFIB, because silicon is the only target material studied computationally, so the variation comes largely from the flux $\phi$. If one considers computational fluxes even lower than the lowest cases shown in Figure 3, the MD calculation becomes prohibitively expensive due to the number of time steps required. But it is certain that the normalized removal rate would remain at $10^{\circ}$, because the potential used here is well fitted to the low flux experimental sputter yield.

In conclusion, we find that FIB material removal, observed over many different target materials, and for varying beam flux, target film thickness, temperature, and other system parameters, is 
subject to a strong erosive-to-thermal transition. A simple dimensionless parameter, $Q_{F I B}$, which we define as the incident power due to the ion beam normalized by the dissipating power due to thermal conduction, predicts this transition. For cases in which $Q_{F I B} \gtrsim 1$, the material removal rate is orders of magnitude faster than in cases of small $Q_{F I B}$. This observation is consistent with all available data reported in the literature, and explains previously anomalous behavior observed for several different materials and FIB experimental configurations. The theory is supported by recent molecular dynamics results on FIB processing of silicon, over a variety of conditions that span two orders of magnitude in the $Q_{F I B}$ parameter. Establishing such a criterion for an erosiveto-thermal material removal transition enables better control and a more complete understanding of the mechanisms of FIB material removal for nanofabrication.

\section{Acknowledgements}

This material is based upon work supported by the Department of Energy, National Nuclear Security Administration, under Award Number(s) DE-NA0002374, and a University of Illinois Computational Science Graduate Fellowship. This work used the Extreme Science and Engineering Discovery Environment (XSEDE), which is supported by National Science Foundation grant number ACI-1053575. 


\section{References}

1. Lugstein A, Basnar B, Smoliner J, Bertagnolli E. FIB processing of silicon in the nanoscale regime. Applied Physics A. 2003;76:545-548.

2. Kolíbal M, Matlocha T, Vystavěl T, Šikola T. Low energy focused ion beam milling of silicon and germanium nanostructures. Nanotechnology. 2011;22:105304.

3. Hopman WC, Ay F, Hu W, et al. Focused ion beam scan routine, dwell time and dose optimizations for submicrometre period planar photonic crystal components and stamps in silicon. Nanotechnology. 2007;18:195305.

4. Li H, Kang D, Blamire M, Huck WT. Focused ion beam fabrication of silicon print masters. Nanotechnology. 2003;14:220.

5. Frey L, Lehrer C, Ryssel H. Nanoscale effects in focused ion beam processing. Applied physics A. 2003;76:1017-1023.

6. Matovic J, Kettle J, Brousseau E, Adamovic N. Patterning of nanomembranes with a focusedion-beam. IEEE; 2008:103-106.

7. Chu D, Wong W, Goodson KE, Pease RFW. Transient temperature measurements of resist heating using nanothermocouples. Journal of Vacuum Science \& Technology B. 2003;21:29852989.

8. Birtcher R, Donnelly S. Plastic flow induced by single ion impacts on gold. Phys Rev Lett. 1996;77:4374.

9. Liu Y, Longo D, Hull R. Ultrarapid nanostructuring of poly (methylmethacrylate) films using Ga focused ion beams. Appl Phys Lett. 2003;82:346-348.

10. Stark T, Shedd G, Vitarelli J, Griffis D, Russell P. H2O enhanced focused ion beam micromachining. Journal of Vacuum Science \& Technology B. 1995;13:2565-2569. 
11. Chu D, Bilir DT, Pease RFW, Goodson KE. Submicron thermocouple measurements of electron-beam resist heating. Journal of Vacuum Science \& Technology B. 2002;20:3044-3046.

12. Das K. Mechanisms of focused ion beam (FIB) material removal and rearrangement at high beam flux. [Ph. D.]. Urbana, IL: University of Illinois at Urbana-Champaign; 2015.

13. Schmied R, Fröch JE, Orthacker A, Hobisch J, Trimmel G, Plank H. A combined approach to predict spatial temperature evolution and its consequences during FIB processing of soft matter. Physical Chemistry Chemical Physics. 2014;16:6153-6158.

14. De La Rubia, T Diaz, Averback R, Benedek R, King W. Role of thermal spikes in energetic displacement cascades. Phys Rev Lett. 1987;59:1930.

15. Orthacker A, Schmied R, Chernev B, et al. Chemical degradation and morphological instabilities during focused ion beam prototyping of polymers. Physical Chemistry Chemical Physics. 2014;16:1658-1666.

16. Das K, Freund JB, Johnson HT. Mechanisms of material removal and mass transport in focused ion beam nanopore formation. Journal of Applied Physics. 2015;117:085304.

17. Gierak J, Madouri A, Biance A, et al. Sub-5nm FIB direct patterning of nanodevices. Microelectronic Engineering. 2007;84:779-783.

18. Das K, Freund J, Johnson H. A FIB induced boiling mechanism for rapid nanopore formation. Nanotechnology. 2014;25:035303. 

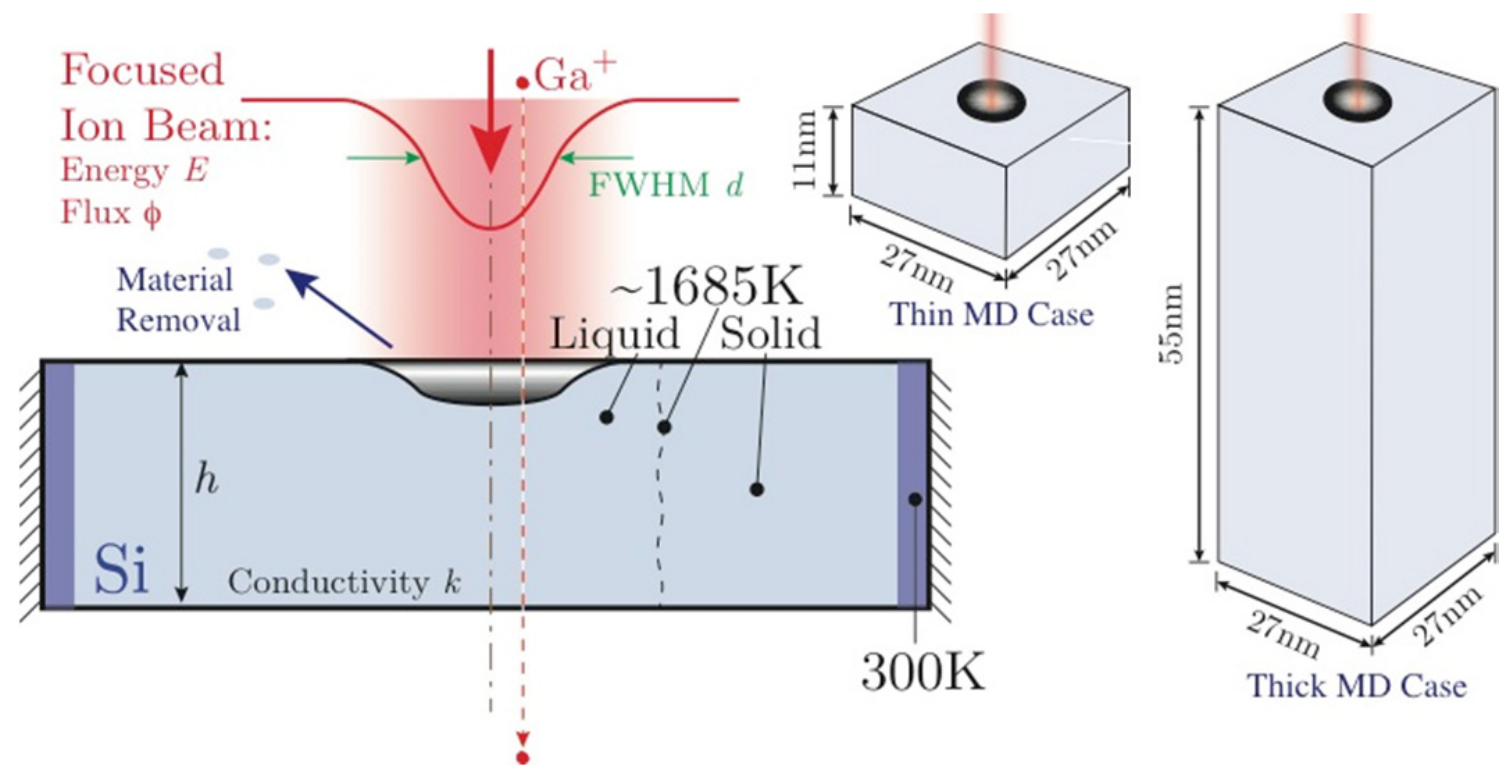

Figure 1. Schematic of the FIB material removal problem. As an example, one may consider a circular $\mathbf{G a}+$ ion beam profile, normally incident on a silicon surface. The beam energy, flux, and diameter, along with the target thickness, thermal conductivity, and temperature difference in the sample, set the scale for either erosive (i.e. mechanical) material removal, or thermally dominated material removal. 


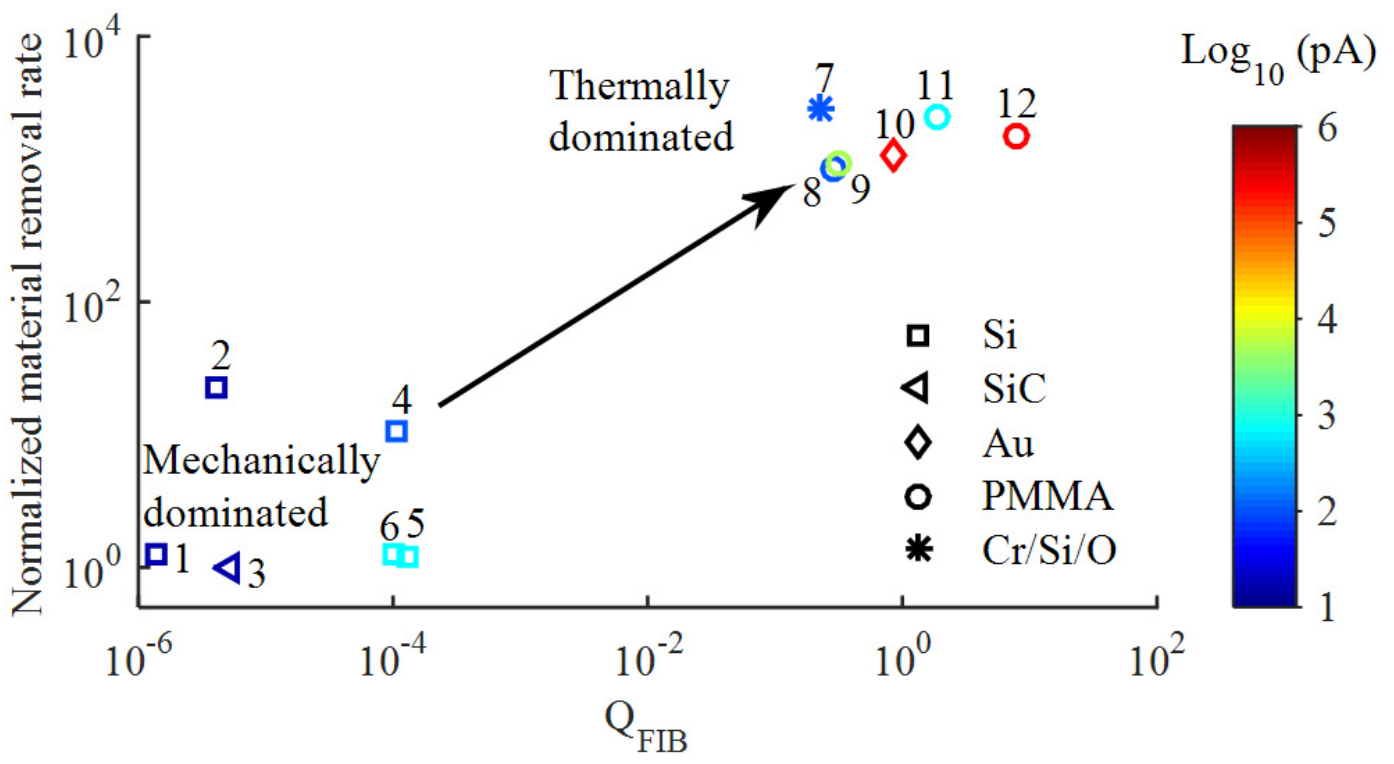

Figure 2. Normalized material removal rate (table I) dependence on $Q_{F I B}$ (table II) experimental. The dimensionless parameter $Q_{F I B}$ marks a clear threshold in material removal behavior. For $Q_{F I B} \sim 1$, material removal occurs significantly faster than sputter erosion theory would predict. The color scale represents beam current, and the symbol shapes indicate materials as labeled. Neither material type, nor beam current, nor any other individual parameter can predict the material removal threshold. The two distinct zones are labeled as "mechanically dominated" and "thermally dominated" regions. The data are from: 1 - Frey et al. ${ }^{5} ; 2$ - Li et al. ${ }^{4} ; 3$ - Gierak et al. ${ }^{17} ; 4$ - Kolibal et al. ${ }^{2} ; 5$ Hopman et al. ${ }^{3} ; 6$ - Lugstein et al. ${ }^{1} ; 7$ - Matovic et al. ${ }^{6} ; 8$ - Liu et al. ${ }^{9}$; 9 - Orthacker et al. ${ }^{15} ; 10$ - Birtcher et al. ${ }^{8} ; 11$ - Liu et al. ${ }^{9}$; and 12 - Chu et al. ${ }^{7,11}$ 


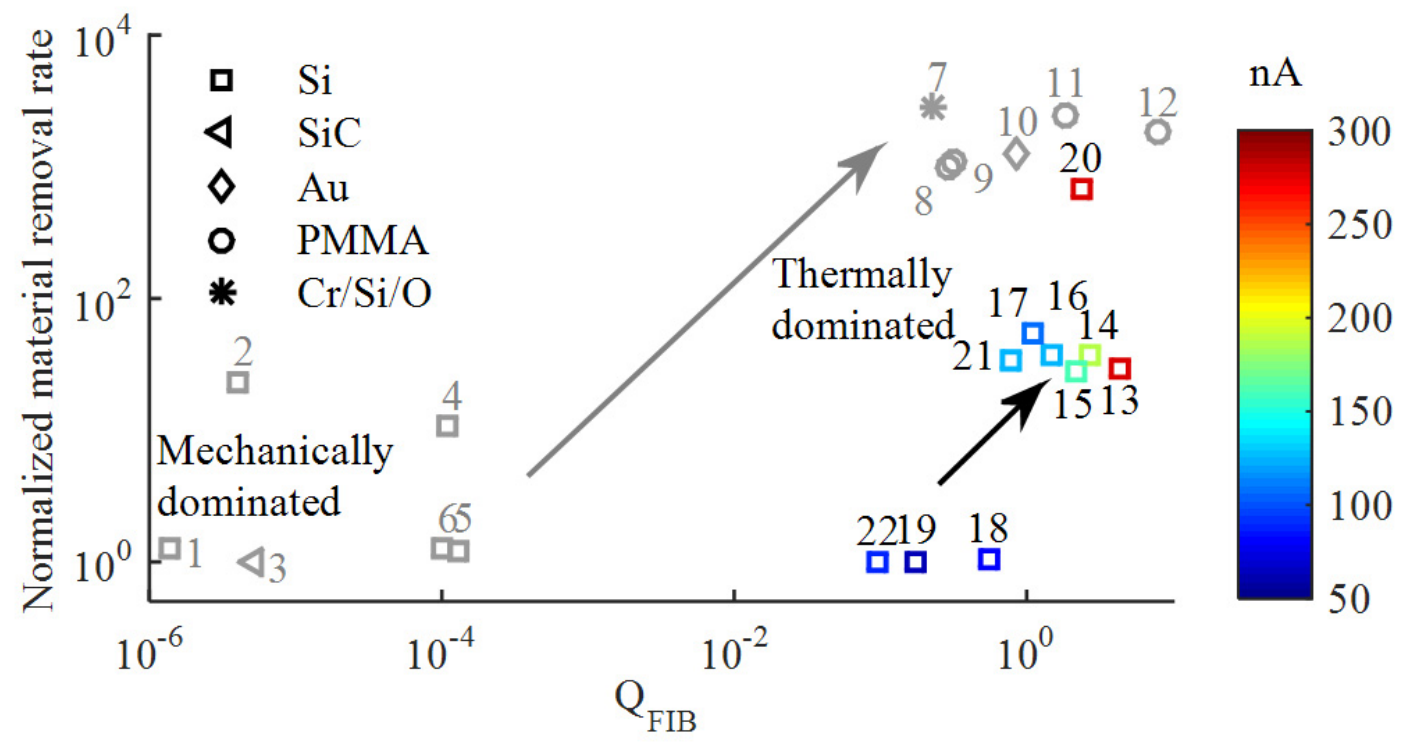

Figure 3. Normalized material removal rate (table I) dependence on $Q_{F I B}$ (table II) computational. The results from the MD simulation are plotted in color. The colorbar represents the beam current in $\mathrm{nA}$, and the symbols represent different materials as shown in the legend. The data plotted here are obtained from a range of sources listed in the caption of Figure 2. Squares labeled 13 through 19 represent thin target cases, and 20 through 22 represent thick target cases. 18, 19, and 22 are below-threshold flux cases. The two distinct zones are labeled as "mechanically dominated" and "thermally dominated" regions. Arrows are added to guide the eye. 
Table I. Normalized material removal rate $\left(R_{M R}\right)$ for previously reported FIB experiments.

\begin{tabular}{|c|c|c|c|c|c|c|c|c|}
\hline Material & $\begin{array}{l}(\mathbf{D}, \mathbf{L}) \text { or }(\mathbf{W}, \mathbf{H}) \\
\quad(\mathbf{n m}, \mathbf{n m}) \\
V_{\text {milled }}=\pi D^{2} L / 4, \\
V_{\text {milled }}=W^{2} H\end{array}$ & $\begin{array}{c}\text { Nominal } \\
\text { Current } \\
(\mathbf{p A}) \\
I\end{array}$ & $\begin{array}{c}\text { Beam } \\
\text { Energy } \\
(\mathrm{keV}) \\
E\end{array}$ & $\begin{array}{c}\text { Dwell } \\
\text { time, } \\
\text { number } \\
\text { of passes } \\
t\end{array}$ & $\begin{array}{c}\begin{array}{c}\text { Ions } \\
\text { delivered }\end{array} \\
N_{d}=\frac{I t}{e}\end{array}$ & $\begin{array}{c}\begin{array}{c}\text { Atoms } \\
\text { removed }\end{array} \\
N_{a}=\frac{V_{\text {milled }}}{V_{\text {atom }}}\end{array}$ & $\begin{array}{c}\begin{array}{c}\text { Ions } \\
\text { required }\end{array} \\
N_{r}=\frac{N_{a}}{S Y}\end{array}$ & $\begin{array}{c}\begin{array}{c}\text { Normalized } \\
\text { material }\end{array} \\
\text { removal rate } \\
R_{M R}=\frac{N_{r}}{N_{d}}\end{array}$ \\
\hline$(\mathrm{Cr} / \mathrm{Si} / \mathrm{O})^{6}$ & 50,10 & 5 & 30 & $0.02 \mathrm{~ms}, 4$ & $2.5 \times 10^{3}$ & $15 \times 10^{6}$ & $7 \times 10^{6}$ & $2.8 \times 10^{3}$ \\
\hline PMMA $^{7}$ & 1700,300 & $150 \times 10^{3}$ & 15 (electron) & $10 \mu \mathrm{s}, 1$ & $9.36 \times 10^{6}$ & $3.4 \times 10^{10}$ & $1.7 \times 10^{10}$ & $1.8 \times 10^{3}$ \\
\hline $\mathbf{S i}^{2}$ & 1000,150 & 1.5 & 30 & $580 \mu \mathrm{s}, 1$ & $355 \times 10^{6}$ & $7.5 \times 10^{9}$ & $3.75 \times 10^{9}$ & 10.6 \\
\hline $\mathbf{S i}^{3}$ & 300,300 & 48 & 30 & $0.1 \mathrm{~ms}, 12$ & $431 \times 10^{6}$ & $1.06 \times 10^{9}$ & $530 \times 10^{6}$ & 1.23 \\
\hline $\mathrm{Si}^{4}$ & 20,20 & 11 & 30 & $100 \mu \mathrm{s}, 1$ & $6.86 \times 10^{3}$ & $314 \times 10^{3}$ & $157 \times 10^{3}$ & 22.9 \\
\hline $\mathrm{Si}^{5}$ & 10000,40 & 150 & 30 & $1 \mu \mathrm{s}, 1$ & $5 \times 10^{10}$ & $1.99 \times 10^{11}$ & $1 \times 10^{11}$ & 2 \\
\hline $\mathrm{Au}^{8}$ & 10,50 & -- & $200(\mathrm{Xe})$ & -- & 1 & $20 \times 10^{3}$ & $10 \times 10^{3}$ & $10 \times 10^{3}$ \\
\hline PMMA $^{9}$ & 60,5 & 1 & 30 & $20 \mu \mathrm{s}, 1$ & \multicolumn{2}{|c|}{$\mathrm{MRR}=1000 \mu \mathrm{m}^{3} / \mathrm{nC}^{9}$} & $=1 \mu \mathrm{m}^{3} / \mathrm{nC}^{10}$ & $1 \times 10^{3}$ \\
\hline
\end{tabular}


Table II. Dimensionless parameter $\boldsymbol{Q}_{F I B}$ for different materials, and processing parameters.

\begin{tabular}{|c|c|c|c|c|c|c|c|c|}
\hline Material & $\begin{array}{c}\text { Energy } \\
E \text { or } V \\
(\mathrm{keV} \text { or } \\
\mathbf{k V})\end{array}$ & $g(h, p)$ & $\begin{array}{l}\text { Current } I \\
\quad(\mathbf{p A})\end{array}$ & $\begin{array}{c}\text { Beam } \\
\text { diameter } \\
d(\mathrm{~nm})\end{array}$ & $\begin{array}{c}\text { Thickness } \\
h(\mathrm{~nm})\end{array}$ & $\begin{array}{c}\text { Thermal } \\
\text { Conductivity } \\
k\left(\mathbf{W m}^{-1} \mathbf{K}^{-1}\right)\end{array}$ & $\begin{array}{c}\text { Temperature } \\
\text { difference } \\
\Delta T=T_{\mathrm{b}}-T_{\mathrm{s}}, \mathrm{K}\end{array}$ & $\mathbf{Q}_{\text {FIB }}$ \\
\hline \multirow{3}{*}{ PMMA $^{9}$} & 30 & 0.17 & 1 & 10 & 5 & 0.01 & 300 & $1.06 \times 10^{-1}$ \\
\hline & 30 & 1 & 11 & 15 & 120 & 0.01 & 300 & $2.92 \times 10^{-1}$ \\
\hline & 30 & 1 & 70 & 23 & 120 & 0.01 & 300 & $1.86 \times 10^{0}$ \\
\hline \multirow{2}{*}{ PMMA $^{7,11}$} & 15 & 1 & $600 \times 10^{3}$ & 2000 & 300 & 0.01 & 300 & $3.18 \times 10^{3}$ \\
\hline & 15 & 1 & $150 \times 10^{3}$ & 1700 & 300 & 0.01 & 300 & $7.96 \times 10^{2}$ \\
\hline \multirow{2}{*}{ PMMA $^{15}$} & 30 & 1 & 500 & 38 & 5000 & 0.01 & 300 & $3.18 \times 10^{-1}$ \\
\hline & 30 & 1 & 100 & 23 & 5000 & 0.01 & 300 & $6.37 \times 10^{-2}$ \\
\hline $\mathrm{Cr} / \mathrm{Si} / \mathbf{O}^{6}$ & 30 & 0.27 & 5 & 16 & 8 & 0.01 & 700 & $2.27 \times 10^{-1}$ \\
\hline $\mathrm{Au}^{8}$ & 200 & 0.25 & $160 \times 10^{3}$ & 10 & 50 & 20 & 2940 & $8.67 \times 10^{-1}$ \\
\hline $\mathbf{S i}^{2}$ & 50 & 1 & 24 & 300 & 150 & 9 & 2600 & $1.09 \times 10^{-4}$ \\
\hline $\mathbf{S i}^{3}$ & 50 & 1 & 48 & 250 & 250 & 9 & 2600 & $1.31 \times 10^{-4}$ \\
\hline $\mathrm{SiC}^{17}$ & 35 & 0.57 & 2 & 5 & 20 & 30 & 4000 & $5.31 \times 10^{-6}$ \\
\hline $\mathbf{S i}^{4}$ & 30 & 1 & 1 & 10 & 100 & 9 & 2600 & $4.08 \times 10^{-6}$ \\
\hline $\mathrm{Si}^{5}$ & 30 & 1 & 1 & 7 & 300 & 9 & 2600 & $1.36 \times 10^{-6}$ \\
\hline $\mathrm{Si}^{1}$ & 50 & 1 & 45 & 68 & 300 & 9 & 2600 & $1.02 \times 10^{-4}$ \\
\hline
\end{tabular}

